\title{
The effectiveness of inhaled Cannabis flower for the treatment of agitation/ irritability, anxiety, and common stress
}

Sarah S. Stith ${ }^{1}$, Xiaoxue Li ${ }^{1}$, Jegason P. Diviant ${ }^{2}$, Franco C. Brockelman ${ }^{3}$, Keenan S. Keeling ${ }^{3}$, Branden Hall ${ }^{3}$ and Jacob M. Vigil ${ }^{2^{*}}$ (D)

\begin{abstract}
Background: An observational research design was used to evaluate which types of commonly labeled Cannabis flower product characteristics are associated with changes in momentary feelings of distress-related symptoms.

Methods: We used data from 2306 patient-directed cannabis administration sessions among 670 people who used the real-time Cannabis effects recording software, Releaf App, between June 6, 2016, and February 23, 2019, for tracking the effects of Cannabis flower consumption. Fixed effects multivariable panel regression techniques were used to establish overall relief by symptom type and to determine which labeled product characteristics (e.g., subspecies/subtype, inhalation method, and major cannabinoid contents) showed the strongest correlation with changes in momentary feelings of agitation/irritability, anxiety, and stress, along with experienced side effects.

Results: In total, a decrease in symptom intensity levels was reported in $95.51 \%$ of Cannabis usage sessions, an increase in $2.32 \%$ of sessions, and no change in $2.16 \%$ of sessions. Fixed effects models showed, on average, respondents recorded a maximum symptom intensity reduction of 4.33 points for agitation/irritability (SE $=0.20$, $p<0.01$ ), 3.47 points for anxiety (SE $=0.13, p<0.01$ ), and 3.98 for stress ( $\mathrm{SE}=0.12, p<0.01$ ) on an 11-point visual analog scale. Fixed effects regressions showed that, controlling for time-invariant user characteristics, mid and high tetrahydrocannabinol (THC) levels were the primary independent predictor of increased symptom relief, and that when broken out by symptom type, this effect was only statistically significant for our largest sample of users, those reporting anxiety rather than agitation/irritability or stress. Cannabidiol (CBD) levels were generally not associated with changes in symptom intensity levels. In a minority of cannabis use sessions $(<13 \%)$, cannabis users reported anxiogenic-related negative side effects (e.g., feeling anxious, irritable, paranoid, rapid pulse, or restless), whereas in a majority of sessions (about 66\%), users reported positive anxiolytic side effects (e.g., feeling chill, comfy, happy, optimistic, peaceful, or relaxed).
\end{abstract}

Conclusions: The findings suggest the majority of patients in our sample experienced relief from distress-related symptoms following consumption of Cannabis flower, and that among product characteristics, higher THC levels were the strongest predictors of relief.

Keywords: Anxiety, Cannabis, Marijuana, Cannabidiol, Tetrahydrocannabinol, Stress, Negative affect

\footnotetext{
*Correspondence: vigilj@unm.edu

${ }^{2}$ Department of Psychology, University of New Mexico, Albuquerque, USA

Full list of author information is available at the end of the article
}

(c) The Author(s). 2020 Open Access This article is licensed under a Creative Commons Attribution 4.0 International License, which permits use, sharing, adaptation, distribution and reproduction in any medium or format, as long as you give appropriate credit to the original author(s) and the source, provide a link to the Creative Commons licence, and indicate if changes were made. The images or other third party material in this article are included in the article's Creative Commons licence, unless indicated otherwise in a credit line to the material. If material is not included in the article's Creative Commons licence and your intended use is not permitted by statutory regulation or exceeds the permitted use, you will need to obtain permission directly from the copyright holder. To view a copy of this licence, visit http://creativecommons.org/licenses/by/4.0/. 


\section{Background}

Americans experience some of the highest levels of stress in the world (Gallup 2019), with over 50\% of recently surveyed adults reporting concerns over issues such as "The future of our nation" (63\%), "money" (62\%), "work" (61\%), "current political environment" (57\%), and "violence and crime" (51\%) according to the American Psychological Association (2017). Stress and anxiety are also among the most common health symptoms for which pharmaceutical medications are prescribed-often for extended periods of time-and are core features of numerous mental and physical health conditions, including depression, addiction, eating disorders, schizophrenia, autism, attention-deficit/hyperactivity disorder, and acute and chronic physical illness and pain (Bandelow et al. 2017; Gureje 2008; De Heer et al. 2014). The most commonly prescribed pharmaceutical medications for symptoms of anxiety include sedatives (e.g., benzodiazepines), antidepressants (SSRIs, SNRIs), antihistamines, and anticonvulsant medicines, with many people also seeking relief through the use of alcohol and illicit drugs (Bandelow and Michaelis 2015; Man et al. 2015; Slee et al. 2019). Alcohol and many conventional psychiatric medications are associated with frequent and severe negative side effects (e.g., addiction and suicidality), adverse reactions, acute toxicity, and even risk of death (Dodds 2017; Kurlawala et al. 2018; MullerOerlinghausen and Berghofer 1999; Read and Williams 2018; Wick 2013).

Stress- and anxiety-related health conditions, particularly post-traumatic stress disorder (PTSD) and chronic pain, are also among the most common health conditions among patients enrolled in state-authorized medical cannabis programs throughout the United States (U.S.) and reasons why people report using and substituting the Cannabis plant for several major classes of medications (e.g., opiates, sedatives, antidepressants) more generally (Piper et al. 2017; Stith et al. 2018a; Stith et al. 2018b; Vigil et al. 2017). According to National Academies of Sciences, E. and M (2017) Committee on the Health Effects of Marijuana, there remains limited clinical evidence that cannabis products offer effective treatment for the improvement of anxiety symptoms, while also acknowledging the scarcity of information regarding routes of administration, dose, efficacy, or side effects of common, commercially available cannabis products in the U.S. This lack of information arises primarily from historical federal regulatory barriers to assessing the Cannabis plant's medicinal potential, which have largely limited investigations to cannabisderived formulates or synthetic analog therapies not widely generalizable to the vast range of common, commercially available products used by millions of people every day (National Academies of Sciences, E. and M
2017; Stith and Vigil 2016). Few studies to date attempt to measure how the broad range of cannabis products, with widely varying cannabinoid contents and ingestion methods, affect momentary symptoms of distress under naturalistic circumstances (Cuttler et al. 2018; Stith et al. 2019; Stith et al. 2018b).

Animal model studies suggest that some of the major cannabinoids (namely cannabidiol (CBD)) have dosedependent biphasic effects (Andrade et al. 2019), exhibiting anxiolytic and antidepressant effects at lower doses (Schier et al. 2014) and anxiogenic responses at higher doses (Kasten et al. 2019). In humans, frequent cannabis use is correlated with higher rates of anxiety disorders, though the direction of causality remains elusive (Crippa et al. 2009; Shalit and Lev-Ran 2020). Retrospective survey data suggests that CBD in particular may be effective for reducing social anxiety and core symptoms of posttraumatic stress disorder (Bonaccorso et al. 2019; Orsolini et al. 2019; Sarris et al. 2020; Van Ameringen et al. 2020). However, there is also increasing interest in the therapeutic value of capitalizing on the synergistic potential of multiple cannabinoids, terpenes, and flavonoids, or what is often described as the "entourage effect" for treatment of anxiety and other mood disorders (Ferber et al. 2019; Russo 2011). Few studies have sought to measure how consumption of Cannabis flower, the most prevalent type of product used in the U.S. (Stith et al. 2019), affects momentary distressrelated symptom levels in real time, along with side effect experiences (e.g., paranoia versus relaxation) that may also contribute to Cannabis' potential anxiogenic or anxiolytic effects.

We analyze one of the largest databases of cannabis user-reported real-time administration sessions in the U.S. for measuring which types of Cannabis flower product characteristics are associated with momentary feelings of distress-related symptom intensity levels and side effect manifestation, taking into account the wide range of characteristics of flower products from cannabinoid content to inhalation method. This research question was operationalized using the mobile software application (app), Releaf App (2019), which was designed for patients to record the types of products, cannabis subtypes or subspecies, cannabinoid contents, consumption methods, and changes in symptom intensity levels and experienced side effects following cannabis consumption, in real time. (Unlike other similar apps, the Releaf App does not incentivize users to enter sessions by rewarding them through earning points towards free products or other forms of compensation.) A previous study using app-based electronically recorded data found that cannabis users report significant reductions in stress following consumption of inhalable cannabis products (e.g., concentrates, oils, and flower) with higher THC and 
CBD levels (Cuttler et al. 2018). However, products such as concentrates (e.g., dabs) often differ from dried natural flower in their representative constituents (e.g., cannabinoid, terpene, and flavonoid contents) and additives (e.g., solvents), and in this previous study, it was unclear how different types of cannabis products affected users (Cuttler et al. 2018). Recent findings have also suggested that the increasing THC can have opposite effects depending on the baseline symptom intensity (Childs et al. 2017).

Rather than including a wide range of formulated and natural cannabis products and treating THC and CBD potency levels (\%/dry wt.) only as continuous measures, we focused exclusively on Cannabis flower and allowed the effects of THC and CBD to vary both linearly and nonlinearly (e.g., low, medium, and high), while controlling for baseline symptom intensity, given that individuals with higher baseline symptom levels have a greater potential for symptom relief, while individuals with lower baseline symptom levels have a greater potential for symptom exacerbation. Furthermore, we accounted for type of strain as marketed (hybrid, sativa, indica), inhalation method (joint, pipe, and vape), session length, and time-invariant user characteristics. This research design enabled us to address the question of which types of commonly labeled Cannabis flower characteristicswithin the restricted number of potency level options in which Cannabis flower "strains" are typically marketed (low, medium, high)-affect changes in distress-related symptom severity. In the current study, people who consumed Cannabis flower for treatment of one of three possible types of distressful (negative affect-related) symptom categories, colloquially phrased "agitation/irritability," "anxiety," or "stress," reported symptom intensity levels immediately prior to and following normative Cannabis consumption and side effects experienced under typical naturalistic circumstances.

\section{Methods \\ Study design}

The study design qualified for exempt status by the University of New Mexico Institutional Review Board, because it posed minimal risk to participants. The Releaf App Privacy Policy, to which users must consent before beginning use, clearly states that anonymized data may be made available to outside researchers. Observational de-identified data subject to an investigator confidentiality agreement were obtained through the owner of the Releaf App ${ }^{\mathrm{Ta}}$, MoreBetter, Ltd. The Releaf App is a publicly available educational software application that is free to download, compatible with both iOS and Android operating systems, and can easily be found through searching the Internet or via websites like cnet. com. In addition, some dispensaries encourage their customers to use the app to help them identify the best cannabis products for their condition. In general, the app is promoted through word of mouth rather than via paid advertising. Real-time session-level effects were recorded by users of the Releaf App. Patients are prompted to indicate their health condition symptom intensity levels on a $0-10$-point visual analog scale, the information that is labeled on the cannabis products they are consuming, and symptom levels and side effects experienced immediately following consumption (Stith et al. 2019; Stith et al. 2018b). More specifically, the app guides the users through a series of screens, first directing users to "select a symptom," then "select cannabis" (i.e., specific product used) and "select equipment" (e.g., joint, pipe, vape), before directing the user to "set symptom level." Once the initial symptom intensity is entered, users can update the symptom level at any time before the end of the session. During an active session, users may also enter optional side effects in response to the questions "How does your mind feel?," "How does your body feel?," "How's your mood?," and "Any other side effects?" before ending and rating the session.

The app includes 50 negative symptoms along with "wellness" that the user can select as the target of their cannabis treatment, with the user capable of treating more than one symptom simultaneously in a session. Out of these 51 options, we selected the three distressrelated symptoms available for selection in the app: agitation/irritability, anxiety, and stress. The app also includes 47 side effects (called "feelings" in the user interface), which the user can report at any time during a session. The available symptoms and side effects were generated through focus groups, by the app developers, and by beta user suggestion. Sessions where patients treated a distress-related symptom were included. Only sessions with baseline symptom intensity levels exceeding zero were included in order to allow for the existence of a treatment effect. We further restricted our sample to symptom levels reported within $4 \mathrm{~h}$ postcannabis consumption, similar to previous investigations (Cuttler et al. 2018; Vigil et al. 2018). In other words, we included only sessions with at least one post-cannabis symptom level reported within $4 \mathrm{~h}$. A total of 23,055 cannabis administration sessions, recorded by 4127 individuals, reported a baseline symptom intensity of one or greater for at least one cannabis administration session used to treat anxiety, agitation/irritability, or stress. We further restricted the sample to include only sessions that reported inhaling dried, natural flower, the most common and homogenous type of cannabis product recorded in the Releaf App data (Stith et al. 2019), leaving 14,693 sessions recorded by 3061 users. Because THC and CBD levels are not mandatory recording, these variables are less commonly reported, and our sample is, 
therefore, further reduced when we restrict the sample to cannabis administration sessions with a full set of product characteristics (subspecies, inhalation method, and THC and CBD levels) reported. We also did not include sessions with THC or CBD levels exceeding 30\%/ dry wt. because levels exceeding $30 \%$ are unlikely to occur naturally in the Cannabis plant. Our THC and CBD measures are not mutually exclusive product categories, but rather track potencies, from 0 to $100 \%$, as voluntarily reported by users, presumably based on product labeling. (Including only sessions with THC and CBD reported potentially biases our sample towards sessions using products purchased from dispensaries. All recreational and medical retail markets in the U.S. require labeled independent potency testing by certified laboratories, but individuals, who may, for example, be home cultivating, are unlikely to have access to the necessary equipment or be willing to pay prices designed for commercial retailers testing large product batches.) The final analysis sample includes 2306 cannabis administration sessions by 670 individuals who recorded at least one user session between June 06, 2016, and February 23,2019 . Among these sessions, $18.3 \%$ reported agitation/irritability, $43.3 \%$ reported anxiety, and 38.4\% reported stress. Side effect reporting is optional, so our side effect analysis is restricted to a sample of 1519 sessions recorded by 559 users.

\section{Study outcomes}

The study outcomes are the change in symptom severity level (symptom relief) and the prevalence of side effects following cannabis consumption. Symptom relief is measured as the minimum symptom severity level within $4 \mathrm{~h}$ minus the baseline symptom intensity. All cannabis sessions in our final sample include at least one symptom update within $4 \mathrm{~h}$ following cannabis consumption with 2.6 ( $\mathrm{SD}=1.8)$ symptom updates in the average session. The resulting symptom relief outcome ranges between 10 (maximum relief) and 9 (maximum exacerbation). In addition to our primary outcome, maximum symptom relief, we also report results for symptom relief within the specified time periods of $1,2,3$, and 4 h, i.e., the last symptom level reported within that time period minus the baseline symptom intensity. To measure the prevalence of side effects, we used dummy variables to indicate if the user reported any of the side effects in the category as well as variables measuring the proportion of total side effects selected by the user within each category.

\section{Statistical analysis}

A multivariable panel regression approach was used to analyze the association between symptom intensity level and cannabis use and the association between product characteristics and symptom relief, controlling for baseline symptom intensity and session length (minutes). To address the concern that symptom intensity changes in response to cannabis reported by the same user are systematically correlated due to individual-specific characteristics, user-specific fixed effects models were used to account for time-invariant user-specific attributes. As such, the effect of cannabis use on symptom intensity level was estimated from a comparison of symptom intensity levels reported by the same user before and after cannabis use. Similarly, the effect of product characteristics on symptom relief was estimated from a comparison across different products by the same user, rather than a comparison across users.

To examine the average effect of cannabis on symptom intensity by symptom type, we regressed symptom intensity levels on a dummy variable equal to one if symptom intensity was reported after cannabis use and equal to zero if reported before cannabis use, controlling for individual fixed effects and running the regressions separately by symptom type.

To explore the effect of product characteristics on symptom relief, we regressed symptom relief on the product characteristics, including THC and CBD content, labeled subtype (hybrid, C. indica, or C. sativa), and inhalation method (joint, pipe, and vaporizer). Our primary THC and CBD measures are the potency from 0 to $30 \% / d r y$ wt. Our plant subspecies variables distinguish between C. indica, C. sativa, and hybrid Cannabis strains. While the colloquial distinction between $C$. indica and $C$. sativa has been widely discounted by the scientific community (Piomelli and Russo 2016), we included these labels because they are still commonly incorporated into Cannabis consumer purchasing decisions. For example, Ontario's government-run online cannabis store differentiates between sativa- and indicadominant strains as does Leafly, the largest aggregator of consumer-friendly cannabis information in the world with more than 100 million visitors each year. We include inhalation method (joint, pipe, or vaporizer) because joints typically are thought to contain lower quality cannabis than loose flower and vaporizing can occur at lower temperatures than combustion via joint or pipe, making controlling for these characteristics potentially important. Our regressions are run for the overall sample and for the three subsamples defined by symptom type. In addition to including product characteristics, we also controlled for session-level precannabis use symptom intensity and session length (minutes up to $4 \mathrm{~h}$-symptom updates beyond $4 \mathrm{~h}$ are not included in our analysis). Baseline symptom intensity is included in all regressions because higher starting symptom levels are associated with greater symptom relief (Vigil et al. 2018). Session length (time from start until 
the last symptom was reported within $4 \mathrm{~h}$ ) is included because the effects of inhaled cannabis may vary systematically with session length. Throughout our regression analyses, standard errors were clustered at the user level and to control for heteroskedasticity and arbitrary correlation among sessions entered by the same user.

In addition to our continuous THC and CBD potency measures, we further explore the relationship between THC, CBD, and symptom relief using categorical THC and $\mathrm{CBD}$ measures to capture nonlinearities in the effect of THC and CBD on symptom relief. We divided our sample fairly evenly into low $\mathrm{THC}=<9 \%$, medium $\mathrm{THC}=10-19 \%$, and high $\mathrm{THC}=20-30 \%$; and low CBD $=0 \%$, medium $\mathrm{THC}=1-9 \%$, and high CBD $=10-30 \%$.

Because we find THC to be a primary driver of symptom relief in the results and it might vary with the other product characteristics, we also test for whether plant subspecies or inhalation method influences the effect of THC on symptom relief, by interacting our continuous measure of THC with those product characteristics. A statistically significant interaction term could arise if, for example, vaporization of cannabis occurs at lower temperatures than combustion of flower in a pipe or joint and this affects THC bioavailability or if joints systematically contain lower grade flower, in which, for example, a greater amount of THC may have already degraded into CBN (cannabinol). We also interact THC with session length to test for variation in the effect of THC over time within $4 \mathrm{~h}$.

We conduct two robustness checks on our symptom relief regression approach. First, because our regression design is inherently based on repeated sessions entered by the same user, we test the robustness of our main results to including only users who entered at least three, four, or five sessions respectively. Second, we extend our time-to-effect analysis by exchanging the maximum symptom relief reported within $4 \mathrm{~h}$ for the difference between baseline symptom intensity and the last symptom level reported within $1,2,3$, and $4 \mathrm{~h}$.

For the side effect outcomes, we use the same regression approach, including the three categories of product characteristics (subtype, inhalation method, and cannabinoid content), baseline symptom intensity level, and session length, along with user fixed effects.

All statistical analyses are conducted using Stata 15.1 (Stata corporation, U.S.).

\section{Results}

Overall, users experienced a symptom intensity reduction in $95.51 \%$ of sessions, no change in symptom intensity in $2.16 \%$ of sessions, and increases in symptom intensity in $2.32 \%$ of sessions. Table 1 presents descriptive statistics for the product characteristics, the starting and minimum symptom severity levels, and the prevalence of side effects. The average cannabis use session involved vaporizing a hybrid strain with at least $10 \% /$ dry wt. THC and 1\%/dry wt. CBD. On average, baseline symptom intensity levels were $5.45(\mathrm{SD}=2.14)$ and minimum post-cannabis symptom intensities were $1.63(\mathrm{SD}=1.8)$, for a mean symptom relief of -3.82 (SD $=3.82$ ). In $63 \%$ of sessions, negative side effects were reported, with positive side effects reported in $97 \%$ of sessions.

Table 2 shows the effect of using inhaled, dried Cannabis flower on reported symptom intensity level using the fixed effects models by symptom type. On average, and as shown in Fig. 1, using an 11-point visual analog scale, respondents recorded a maximum symptom intensity reduction of 3.82 points $(\mathrm{SE}=0.11, p<0.01)$ in the overall distress-related symptom sample, 4.33 points in sessions treating specifically agitation/irritability (SE = $0.20, p<0.01$ ), 3.47 points in those treating anxiety (SE $=0.13, p<0.01$ ), and 3.98 points in those treating stress $(\mathrm{SE}=0.12, p<0.01)$.

Table 3 shows the category, prevalence, and average symptom relief across each side effect ordered by frequency. Of the 47 possible side effects (17 negative, 19 positive, and 11 context-specific), the least commonly reported were the negative side effects (e.g., paranoid [4\%] and experiencing a rapid pulse [3\%]) and the most commonly reported were the positive side effects (e.g., relaxed [66\%] and feeling peaceful [57\%]), with the context-specific side effects falling in between (e.g., feeling high [47\%] and thirsty [27\%]). Significant changes in symptom were coreported with each of the side effect experiences.

Table 4 presents the results for the association between product characteristics and symptom relief in our overall sample and by symptom subgroup, treating THC and CBD levels as continuous variables. In column 1, the results show that, among product characteristics, only THC affects symptom relief-maximum symptom relief improves by 0.02 points for every one percentage point increase in THC. Comparing across columns shows that this effect is driven by sessions treating anxiety and stress rather than agitation/irritability. Longer sessions and sessions with higher starting symptoms are associated with greater symptom relief overall and across symptom types, as will be shown consistently across tables.

Table 5 uses the categorical THC and CBD measures to evaluate general nonlinearities in the effects of these variables. As shown in column 1, both ranges of THC levels above $10 \%$ are associated with greater symptom relief. In other words, THC potencies above $10 \%$ offer more relief than those below, but even higher levels of THC do not, e.g., THC levels of $20-30 \%$ offer the same amount of relief based on $F$ tests of the difference 
Table 1 Session characteristics, maximum symptom relief, and side effects when using inhaled, dried Cannabis flower

\begin{tabular}{|c|c|c|c|c|}
\hline & $\%$ or mean & $N$ or Std. Dev. & Minimum & Maximum \\
\hline \multicolumn{5}{|c|}{ Panel A: THC (2306 symptom-sessions, 670 users) } \\
\hline$\% / d r y$ wt. THC & 17.27 & $(7.51)$ & 0 & 30 \\
\hline $\mathrm{THC}<10 \%$ & $21 \%$ & 476 & 0 & 1 \\
\hline THC 10-19\% & $39 \%$ & 907 & 0 & 1 \\
\hline THC 20-30\% & $40 \%$ & 923 & 0 & 1 \\
\hline \multicolumn{5}{|c|}{ Panel B: CBD (2306 symptom-sessions, 670 users) } \\
\hline \%/dry wt. CBD & 6.27 & $(6.30)$ & 0 & 29 \\
\hline $\mathrm{CBD}<1 \%$ & $23 \%$ & 528 & 0 & 1 \\
\hline CBD 1-9\% & $45 \%$ & 1049 & 0 & 1 \\
\hline CBD $10-30 \%$ & $32 \%$ & 729 & 0 & 1 \\
\hline \multicolumn{5}{|c|}{ Panel C: subspecies (2306 symptom-sessions, 670 users) } \\
\hline Hybrid & $53 \%$ & 1230 & 0 & 1 \\
\hline C. indica & $27 \%$ & 617 & 0 & 1 \\
\hline C. sativa & $20 \%$ & 459 & 0 & 1 \\
\hline \multicolumn{5}{|c|}{ Panel D: inhalation method (2306 symptom-sessions, 670 users) } \\
\hline Joint & $17 \%$ & 390 & 0 & 1 \\
\hline Pipe & $40 \%$ & 927 & 0 & 1 \\
\hline Vape & $43 \%$ & 989 & 0 & 1 \\
\hline \multicolumn{5}{|c|}{ Panel E: outcome and control variables (2306 symptom-sessions, 670 users) } \\
\hline Baseline symptom intensity & 5.45 & $(2.14)$ & -10 & 0 \\
\hline Minimum symptom intensity & 1.63 & $(1.80)$ & 1 & 10 \\
\hline Symptom change & -3.82 & $(2.16)$ & 0 & 10 \\
\hline \multicolumn{5}{|c|}{ Panel F: side effects (1519 sessions, 559 users) } \\
\hline Any negative side effect & $63 \%$ & 954.00 & 0 & 1 \\
\hline$\%$ of negative side effects & $10 \%$ & $(0.12)$ & 0 & 0.82 \\
\hline Any positive side effect & $97 \%$ & 1471.00 & 0 & 1 \\
\hline$\%$ of positive side effects & $28 \%$ & $(0.18)$ & 0 & 1 \\
\hline Any context-specific side effect & $81 \%$ & 1234.00 & 0 & 1 \\
\hline$\%$ of context-specific side effects & $19 \%$ & $(0.16)$ & 0 & 0.91 \\
\hline
\end{tabular}

Our dichotomous variables are measured $\{0,1\}$ and are reported in the tables as percentages ranging from 0 to 100 , along with the number of sessions reporting "1." Our nondichotomous variables, \%/dry wt. tetrahydrocannabinol (THC), \%/dry wt. cannabidiol (CBD), baseline (pre-consumption) symptom intensity, minimum (post-consumption) symptom intensity, symptom change (minimum post-consumption symptom level minus starting symptom intensity), \% of negative side effects, \% of positive side effects, and percent of context-specific side effects, range in value as reported above with standard deviations reported in parentheses. The overall sample includes distress-related symptoms available for selection through the app: agitation/irritability, anxiety, and stress. Nineteen positive, seventeen negative, and eleven context-specific side effects were available for selection

Table 2 Fixed effects model for use of inhaled, dried Cannabis flower on reported symptom intensity level by reported symptom

\begin{tabular}{lllll}
\hline & $\mathbf{( 1 )}$ & $\mathbf{( 2 )}$ & $\mathbf{( 3 )}$ & $\mathbf{( 4 )}$ \\
& All & Agitation/irritability & Anxiety & Stress \\
\hline Post-cannabis use & $-3.824^{* * *}(0.110)$ & $-4.332^{* * *}(0.198)$ & $-3.474^{* * *}(0.125)$ & $-3.977^{* * *}(0.122)$ \\
Constant & $5.452^{* * *}(0.055)$ & $5.754^{* * *}(0.099)$ & $5.246^{* * *}(0.062)$ & $5.540^{* * *}(0.061)$ \\
Observations & 4612 & 844 & 1996 & 1772 \\
$R$-squared & 0.644 & 0.707 & 0.635 & 0.686 \\
Number of users & 670 & 206 & 441 & 360
\end{tabular}

Each column represents a separate regression. The outcome is the reported symptom level. The explanatory variable is a dummy variable that equals to one if symptom level is reported after cannabis use and equals zero if reported before cannabis use. Models are estimated using an individual fixed effects model. Standard errors, clustered at the individual user level, are shown in parentheses

${ }^{* * *} p<0.001$ 


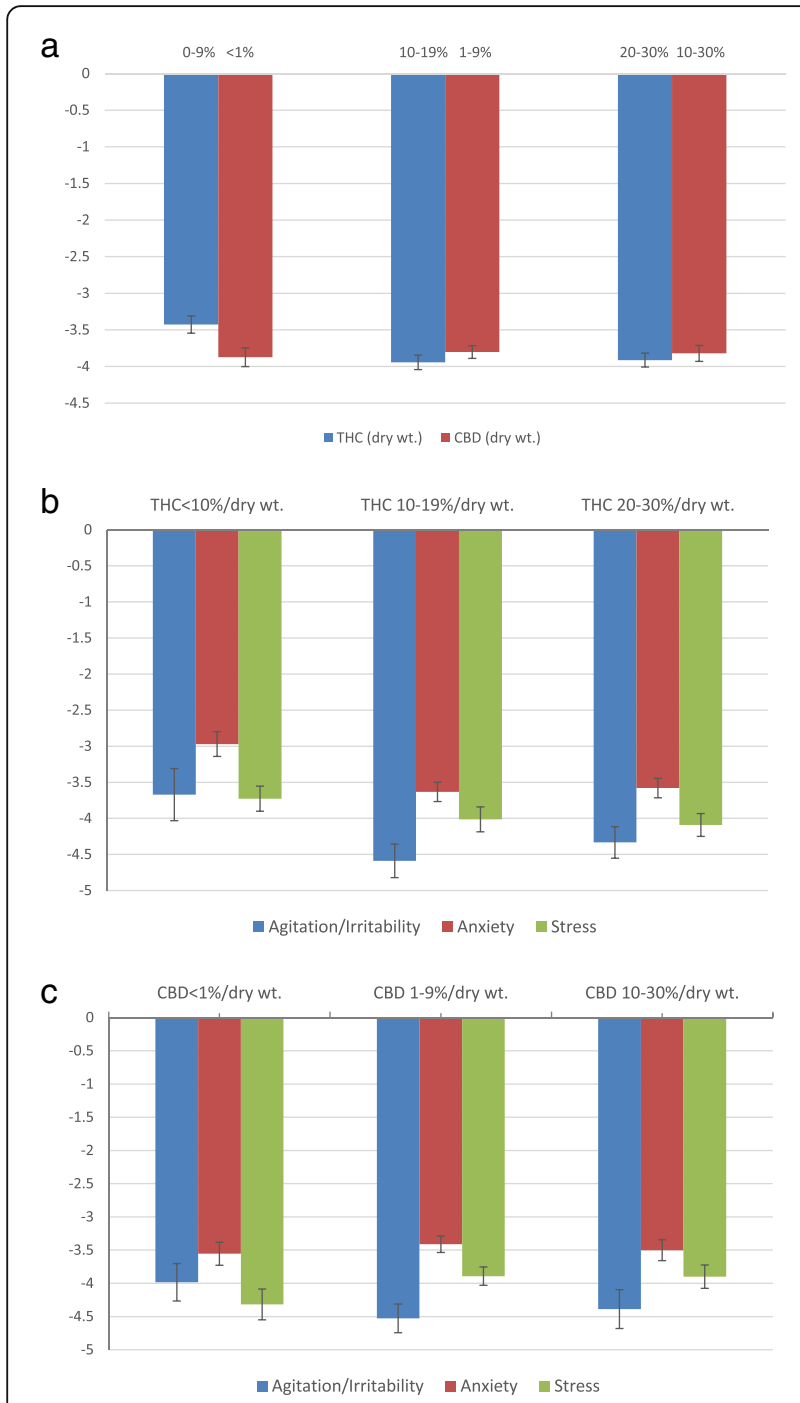

Fig. 1 Maximum symptom relief when using inhaled, dried Cannabis flower by THC and CBD levels. a Symptom relief by CBD and THC level in overall sample. $\mathbf{b}$ Symptom relief by THC level by symptom type. c Symptom relief by CBD level by symptom type. Notes: Adjusted maximum symptom relief is reported, which refers to covariate-adjusted change in symptom severity (minimum symptom level reported within $4 \mathrm{~h}$ after session initiation minus the starting symptom level) and was obtained from a user-level fixed effects model controlling for subtype, inhalation method, and starting symptom level. Tetrahydrocannabinol (THC) and cannabidiol (CBD) are measured in \%/dry wt. CBD categories are controlled for in the THC figure and THC categories are controlled for in the CBD figure. Potency levels represent percentage of labeled, laboratory-tested dried weight

between the coefficients. The results using categorical measures of THC and CBD indicate that the effect is driven by sessions treating anxiety. Small sample sizes could be a factor in the insignificance of the coefficients in column 2. As shown in column 1, the results in this table also offer suggestive evidence that strains labeled as C. sativa might offer less relief from distress-related symptoms than those labeled as C. indica or hybrid strains, but the effect is not significant in the analyses by specific symptom.

Interactions between $\mathrm{THC}$, the other product characteristics, and session length are presented in Supplemental Table 1. None of the coefficients on the interaction terms is statistically significant, implying that the effects of THC on relief from distress-related symptoms do not vary with the plant subspecies, the method of inhalation, or the session length.

Supplemental Tables 2 and 3 report the results from our robustness checks. In Supplemental Table 2, the effect of THC does not appear to vary depending on the total number of sessions entered by a user. Suggestive evidence of decreased symptom relief from strains labeled as $C$. sativa appears again in this table. Supplemental Table 3 further supports this same story. In Supplemental Table 3, our outcome variable is now the difference between the last symptom intensity reported within the specified time period and the baseline symptom intensity rather than the difference between the minimum symptom intensity reported within $4 \mathrm{~h}$ and the baseline symptom intensity level. Again, a one percentage point increase in THC is associated with a 0.02 point improvement in symptom relief. This table provides the strongest support for the potential that strains labeled as $C$. sativa offer less symptom relief.

Supplemental Table 4 presents our results for the effects of our independent variables on the percent of each of these side effect categories reported. None of the product characteristics or session length appears to affect negative side effect reporting. Evidence exists in columns 2 and 3 that THC increases the likelihood of reporting positive or context-specific side effects. Lastly, C. indica decrease the likelihood of experiencing positive side effects.

\section{Discussion}

Feelings of distress reflect a basic dimension of human emotionality that is expressed under conditions when the individual perceives a lack of control over threatening environmental pressures and/or forces (Buchanan 2000; Gallagher et al. 2014; Vigil 2009), and it is possible that Cannabis usage reduces such perceptions. The current study helps explain why many patients attempting to treat feelings of distress voluntarily substitute medical cannabis for several classes of prescription medications, including those used to treat negative affect (e.g., SSRIs, SNRIs, TCAs, MAOIs, beta blockers, atypical antipsychotics, and benzodiazepines), when given the legal opportunity to do so (Bachhuber et al. 2014; Bradford and Bradford 2016; Piper et al. 2017; Powell et al. 2018; Stith et al. 2018a; Vigil et al. 2017; Wen and Hockenberry 2018). Expanding upon a previous study 
Table 3 Session side effect frequencies and maximum symptom relief when using inhaled, dried Cannabis flower

\begin{tabular}{|c|c|c|c|}
\hline Side effect & $\%$ session reporting & Symptom relief & Category \\
\hline Relaxed & $66 \%$ & -3.96 & Positive \\
\hline Peaceful & $57 \%$ & -3.99 & Positive \\
\hline Chill & $47 \%$ & -3.83 & Positive \\
\hline High & $47 \%$ & -3.79 & Context-specific \\
\hline Comfy & $41 \%$ & -4.00 & Positive \\
\hline Dreamy & $32 \%$ & -3.84 & Positive \\
\hline Happy & $29 \%$ & -4.20 & Positive \\
\hline Clear & $29 \%$ & -3.80 & Positive \\
\hline Reflective & $29 \%$ & -4.12 & Positive \\
\hline Focused & $28 \%$ & -3.98 & Positive \\
\hline Tuned & $27 \%$ & -4.01 & Positive \\
\hline Light & $27 \%$ & -4.17 & Positive \\
\hline Thirsty & $27 \%$ & -4.13 & Context-specific \\
\hline Tingly & $24 \%$ & -4.16 & Context-specific \\
\hline Dry mouth & $21 \%$ & -4.17 & Negative \\
\hline Great & $21 \%$ & -4.31 & Positive \\
\hline Sleepy & $20 \%$ & -3.94 & Context-specific \\
\hline Optimistic & $20 \%$ & -4.32 & Positive \\
\hline Foggy & $20 \%$ & -3.80 & Negative \\
\hline Hungry & $19 \%$ & -3.87 & Context-specific \\
\hline Grateful & $19 \%$ & -4.18 & Positive \\
\hline Scattered & $18 \%$ & -4.19 & Negative \\
\hline Thinky & $16 \%$ & -3.97 & Context-specific \\
\hline Productive & $16 \%$ & -3.92 & Positive \\
\hline Couchlocked & $16 \%$ & -4.12 & Context-specific \\
\hline Distracted & $15 \%$ & -4.14 & Context-specific \\
\hline Unmotivated & $14 \%$ & -3.98 & Negative \\
\hline Forgetful & $13 \%$ & -4.14 & Negative \\
\hline Energetic & $12 \%$ & -3.95 & Positive \\
\hline Creative & $12 \%$ & -4.17 & Positive \\
\hline Restless & $12 \%$ & -4.01 & Negative \\
\hline Talkative & $11 \%$ & -4.09 & Context-specific \\
\hline Coughing & $11 \%$ & -4.08 & Negative \\
\hline Anxious & $10 \%$ & -3.66 & Negative \\
\hline Red eyes & $9 \%$ & -4.22 & Negative \\
\hline Frisky & $9 \%$ & -4.33 & Positive \\
\hline Silly & $9 \%$ & -4.10 & Context-specific \\
\hline Irritable & $8 \%$ & -4.23 & Negative \\
\hline Active & $8 \%$ & -3.98 & Positive \\
\hline Dizzy & $8 \%$ & -3.60 & Negative \\
\hline Headache & $6 \%$ & -3.71 & Negative \\
\hline Confused & $6 \%$ & -3.41 & Negative \\
\hline Paranoid & $4 \%$ & -3.09 & Negative \\
\hline Visuals & $4 \%$ & -4.33 & Context-specific \\
\hline
\end{tabular}


Table 3 Session side effect frequencies and maximum symptom relief when using inhaled, dried Cannabis flower (Continued)

\begin{tabular}{llll}
\hline Side effect & \% session reporting & Symptom relief & Category \\
\hline Rapid pulse & $3 \%$ & -3.67 & Negative \\
Clumsy & $3 \%$ & -4.21 & Negative \\
Nausea & $1 \%$ & -3.00 & Negative \\
\hline
\end{tabular}

Table 3 reports the percent of sessions in which the side effect was recorded as well as the average symptom relief experienced in those sessions. We categorize the side effects as negative, context-specific, or positive. Side effects were reported in $65.7 \%(N=1519)$ of the 2306 sessions in the sample

(Cuttler et al. 2018), our real-time effects showed that Cannabis flower is an effective anxiolytic medication and it is relatively fast-acting, but it can also produce negative side effects that may exacerbate momentary symptoms of negative affect in a small minority of sessions. While in some sessions users reported no change in symptom intensity levels or experiencing feelings that could contribute to distress (e.g., feeling restless), in 95\% of sessions, people reported an average overall symptom intensity reduction of approximately 3.8 points on a standard 0 to 10 visual analog scale.

The current observational research design maximizes the external validity and generalizability of the findings through assessments of patients' actual medical treatment decisions, including their choice across a range of product options, and the experienced effects of those decisions, in real-time. The mobile software technology used in the study solves the significant practical, medical, and scientific challenge of monitoring and measuring therapeutic and side effects across the vast range of products available at medical and recreational cannabis dispensaries, which vary by strains, consumption method, and major cannabinoid contents. The current results suggest benefits from patient-directed cannabis therapy as a mid-level anxiolytic treatment. Thus, despite the conventional wisdom that smoking cannabis makes one paranoid, we found consumption much more likely to be associated with relaxation and sense of calm, with users most likely to report feelings of peacefulness, optimism, and happiness. One potential explanation for the disparity between our findings and popular perceptions of cannabis is that the "paranoia" users may have historically reported could have arisen in part from cannabis' illicit status (e.g., anxiety over committing an illegal act), rather than the plant's typical endemic pharmacodynamic effects when consumed in contexts typical of legal medicinal use. Individual factors such as user's experience level likely also contribute to cannabis' effects.

This study finds that the effectiveness and side effect manifestation vary with the characteristics of the Cannabis flower consumed and the specific type of distressrelated symptom treated. In particular, mid to higher THC levels are statistically significant predictors of increased symptom relief, while CBD levels and inhalation method (joint, pipe, vape) are largely not. In contrast, plants labeled as C. sativa were associated with less overall symptom relief. The relationship between higher

Table 4 Session product characteristics' effects on symptom relief when using inhaled, dried Cannabis flower and treating cannabinoid measurements as continuous variables

\begin{tabular}{lllll}
\hline & Overall Sample & Agitation/irritability & Anxiety & Stress \\
& $(\mathbf{1})$ & $\mathbf{( 2 )}$ & $\mathbf{( 3 )}$ & $\mathbf{( 4 )}$ \\
\hline THC (\%/dry wt.) & $-0.020^{* * *}(0.006)$ & $0.006(0.018)$ & $-0.024^{* *}(0.008)$ & $-0.021^{*}(0.009)$ \\
CBD (\%/dry wt.) & $-0.002(0.009)$ & $0.012(0.015)$ & $-0.001(0.010)$ & $-0.001(0.012)$ \\
C. indica & $-0.014(0.104)$ & $-0.209(0.215)$ & $0.033(0.133)$ & $0.018(0.169)$ \\
C. sativa & $0.215(0.111)$ & $0.162(0.209)$ & $0.222(0.127)$ & $0.250(0.166)$ \\
Pipe & $0.093(0.256)$ & $0.499(0.653)$ & $-0.385(0.213)$ & $0.498(0.409)$ \\
Vape & $0.135(0.274)$ & $0.708(0.664)$ & $-0.222(0.268)$ & $0.492(0.406)$ \\
Session length (min) & $-0.007^{* * *}(0.001)$ & $-0.005^{*}(0.002)$ & $-0.007^{* * *}(0.001)$ & $-0.008^{* * *}(0.001)$ \\
Baseline symptom intensity & $-0.662^{* * *}(0.040)$ & $-0.694^{* * *}(0.071)$ & $-0.608^{* * *}(0.069)$ & $-0.669^{* * *}(0.052)$ \\
Constant & $0.281(0.296)$ & $-0.801(0.744)$ & $0.639(0.379)$ & $-0.058(0.437)$ \\
Number of sessions & 2306 & 422 & 998 & 441 \\
Number of users & 670 & 206 & 886 & 360 \\
\hline
\end{tabular}

Each column represents a separate regression. The outcome is the difference between the lowest reported symptom level within $4 \mathrm{~h}$ of initiating the session and the starting symptom level. The first column reports results for the whole sample, while columns 2 to 4 distinguish between specific distress-related symptoms reported. C. indica and C. sativa are relative to hybrid strains, and pipe and vape are relative to joint. All regressions are estimated using a fixed effects model and control for session length and baseline symptom intensity. Standard errors, clustered at the individual user level, are shown in parentheses

${ }^{* * *} p<0.001,{ }^{* *} p<0.01,{ }^{*} p<0.05$ 
Table 5 Session product characteristics' effects on symptom relief when using inhaled, dried Cannabis flower and treating cannabinoid measurements as categorical variables

\begin{tabular}{|c|c|c|c|c|}
\hline & $\begin{array}{l}\text { Overall sample } \\
\text { (1) }\end{array}$ & $\begin{array}{l}\text { Agitation/irritability } \\
\text { (2) }\end{array}$ & $\begin{array}{l}\text { Anxiety } \\
\text { (3) }\end{array}$ & $\begin{array}{l}\text { Stress } \\
(4)\end{array}$ \\
\hline THC 10-19\%/dry wt. & $-0.377^{* *}(0.123)$ & $-0.472(0.469)$ & $-0.618^{* * *}(0.170)$ & $-0.024(0.167)$ \\
\hline THC 20-30\%/dry wt. & $-0.345^{* *}(0.117)$ & $-0.131(0.452)$ & $-0.599^{* * *}(0.165)$ & $0.214(0.162)$ \\
\hline CBD 1-9\%/dry wt. & $0.007(0.138)$ & $-0.333(0.217)$ & $0.120(0.152)$ & $0.518(0.411)$ \\
\hline CBD 10-30\%/dry wt. & $-0.057(0.188)$ & $-0.270(0.292)$ & $-0.051(0.199)$ & $0.530(0.409)$ \\
\hline C. indica & $-0.024(0.106)$ & $-0.163(0.200)$ & $0.037(0.141)$ & $-0.110(0.174)$ \\
\hline C. sativa & $0.212^{*}(0.104)$ & $0.068(0.243)$ & $0.231(0.131)$ & $-0.227(0.171)$ \\
\hline Pipe & $0.079(0.260)$ & $0.418(0.646)$ & $-0.41(0.220)$ & $0.116(0.265)$ \\
\hline Vape & $0.146(0.274)$ & $0.634(0.672)$ & $-0.229(0.257)$ & $0.089(0.313)$ \\
\hline Session length (min) & $-0.007^{* * *}(0.001)$ & $-0.005^{*}(0.002)$ & $-0.007^{* * *}(0.001)$ & $-0.008^{* * *}(0.001)$ \\
\hline Baseline symptom intensity & $-0.661^{* * *}(0.039)$ & $-0.693^{* * *}(0.072)$ & $-0.604^{* * *}(0.067)$ & $-0.667^{* * *}(0.052)$ \\
\hline Constant & $0.219(0.300)$ & $-0.105(0.848)$ & $0.649(0.350)$ & $-0.402(0.478)$ \\
\hline Number of sessions & 2306 & 422 & 998 & 886 \\
\hline Number of users & 670 & 206 & 441 & 360 \\
\hline
\end{tabular}

Each column represents a separate regression. The outcome is the difference between the lowest reported symptom level within $4 \mathrm{~h}$ of initiating the session and the starting symptom level. The first column reports results for the whole sample, while columns 2 to 4 distinguish between specific distress-related symptoms reported. The omitted category for the THC categories is THC less than $10 \%$ and for the CBD categories is CBD equal to $0 \%$. C. indica and C. sativa are relative to hybrid strains, and pipe and vape are relative to joint. All regressions are estimated using a fixed effects model and control for session length and baseline symptom intensity. Standard errors, clustered at the individual user level, are shown in parentheses

${ }^{* * *} p<0.001,{ }^{* *} p<0.01,{ }^{*} p<0.05$

THC and increased symptom relief appears to be driven by cannabis sessions treating specifically "anxiety" and to a lesser extent "stress" rather than "agitation/irritability," although sample sizes are small in the subgroup analyses and some of the variation could arise from anxiety being a more clinical and clearly defined term than the other two symptoms included in our analyses.

The differences in symptom relief across THC levels might arise because THC has been shown to both decrease and increase negative mood states. However, unlike in this study, smaller doses of isolated or synthetic THC have been found to be anxiolytic and higher doses (in isolated form) appeared to be anxiogenic (Childs et al. 2017). The mechanisms by which THC potency levels can produce these biphasic effects are not fully understood and likely encompass multiple brain regions and complex interactions with other chemotypic characteristics of the plant and endogenous neurotransmitters. In rats, low doses of THC microinjected into the prefrontal cortex (e.g., $10 \mu \mathrm{g})$ and ventral hippocampus (e.g., $5 \mu \mathrm{g}$ ) were anxiolytic, whereas higher doses appeared to be anxiogenic. By contrast, microinjections of low doses (e.g., $1 \mu \mathrm{g}$ ) of THC in the basolateral amygdala produced anxiogenic effects, while higher doses were found to be ineffective (Rubino et al. 2008). Moreover, while low doses of THC stimulate an anxiogenic signal in the amygdala, the anxiolytic signals generated in the prefrontal cortex and hippocampus override this effect by suppressing the amygdala activation (Rubino et al. 2008).
THC's anxiolytic effects are likely mediated by $\mathrm{CB}_{1}$ and $\mathrm{CB}_{2}$ receptors, whose respective roles appear to be to modulate neurotransmitter and cytokine release (Pertwee and Ross 2002). For example, both $\mathrm{CB} 1$ and $5-\mathrm{HT}_{2 \mathrm{~A}}$ receptors are expressed in most glutamatergic neurons in the prefrontal cortex and hippocampus (Hill et al. 2007). The $\mathrm{CB}_{1}$ and $5-\mathrm{HT}_{2 \mathrm{~A}}$ receptors have been shown to physically interact and form heteromers, and the costimulation of these $\mathrm{CB}_{1} \mathrm{R}-5-\mathrm{HT}_{2 \mathrm{~A}} \mathrm{R}$ heteromers appears to modulate cellular signaling in specific brain structures, including the prefrontal cortex and the hippocampus (Viñals et al. 2015). Other research suggests that anxiolytic effects of THC are mediated through the $\mathrm{CB}_{1}$ receptors on cortical glutamatergic terminals (Rey et al. 2012). Hence, $C_{1} R-5-H_{2} T_{2 A} R$ heterodimerization may play a significant role in the reduction of glutamate levels in the prefrontal cortex and hippocampus, which could be leading the reductions in visceral feelings of distress reported by app users. However, in addition to the complexity of understanding the effects of isolated THC on the brain, the synergistic effects of THC and other compounds in the cannabis plant, including CBD, are even less understood. The fact that higher THC appears to confer greater anxiolytic effects in our study at higher THC levels suggests that the whole natural Cannabis plant may act very differently on the brain as compared to synthetic or derived THC isolates.

Although we were unable to account for these in our study, terpene and terpenoid contents that contribute to 
the overall phytocannabinoid-terpene-terpenoid synergy or "entourage effect" from whole, natural Cannabis plants can vary from one plant to another and by inhalation method. Many terpenes and terpenoids share a direct precursor with phytocannabinoids. For example, geranyl pyrophosphate is a precursor to the phytocannabinoids found in Cannabis and to the monoterpenes and monoterpenoids. Terpenes and terpenoids may comprise as much as $10 \%$ of total cannabis trichome content, and individual concentrations of $>500 \mathrm{ppm}$ are considered to be of pharmacological interest. Serum terpene levels in the single-digit $\mathrm{ng} \mathrm{mL} \mathrm{m}^{-1}$ range have been found to induce physiological effects potent enough to alter animal and human behavior, including anxiolytic and perceived negative side effects (Ross 2003; Russo 2011; SoutoMaior et al. 2011). For example, linalool and limonene have both demonstrated in several studies to possess potent anxiolytic properties (Carvalho-Freitas and Costa 2002; De Almeida et al. 2014; De Moraes Pultrini et al. 2006; Franco et al. 2016; Harada et al. 2018; Lima et al. 2013; Linck et al. 2010; Souto-Maior et al. 2011). $\beta$ Caryophyllene is a selective full agonist at the $\mathrm{CB}_{2}$ receptor and has unique effects on negative affect, making the $\mathrm{CB}_{2}$ receptor a prospective therapeutic target for the treatment of both anxiety and depression with cannabis (Bahi et al. 2014; Galdino et al. 2012; Kamal et al. 2018; Russo 2011). Future studies identifying strains with the most notable effects on negative affect should help elucidate why we find suggestive evidence that products labeled as "indica" and "hybrid" may be to be more anxiolytic than strains typically labeled as "sativa." Future research will benefit from identifying and measuring the effects of particulate chemotypic profiles, including cannabinoid-terpene-flavonoid combinations, magnitudes, and ratios across varying plant strains, beyond conventional plant characteristic labeling.

The current study does have limitations, the most prominent of course being the lack of absolute experimental control (e.g., double-blinded randomization and use of a placebo intervention) and the analysis of naturalistic behaviors and dosage patterns rather than a directed and uniform regimen. Likewise, the study did not include individuals who do not use cannabis to treat their distress or any cannabis consumption sessions not tracked in the app potentially resulting in selection bias. People who choose to use cannabis to treat their distress-related symptoms may be those most likely to benefit from it or those for whom conventional treatments are less effective. The direction of the bias for app use is not as clear. Not using the app could be simply a matter of not knowing about the app or a dislike of app-based technologies, or, along with attrition, be due to dissatisfaction with cannabis or the app. Alternatively, not using the app or stopping app use could arise from satisfaction with existing cannabis use and the lack of a need to explore other product options. Within the app itself, the overt pro-cannabis language would also likely influence the type of individuals who would use the app and attract users with views aligned with the authors of the app; similar types of sample selection biases are common in large epidemiological studies where people volunteer their time to discuss or describe a discrete research topic and, in clinical trials, among people that choose to be participants in a study. Although our study extended the literature by incorporating a wider range of product characteristics than has been previously examined, we still were not able to include the full range of characteristics of products available (e.g., terpene profiles) and did not include nonflower cannabis products. We also did not account for user demographics, cannabis experience, or the concomitant use of medications other than cannabis beyond those time-invariant characteristics captured by the user fixed effects. Additional factors such as frequency of use and resultant changes in tolerance levels likely also contribute to individual differences in potential anxiogenic and anxiolytic effects, and future research may benefit by incorporating dosage and tolerance-related factors in their analyses. Finally, while improvements in testing and regulatory oversight may be reducing this issue, studies have shown that THC and CBD levels reported on product labels are often inaccurate, particularly at the higher end of the distribution, which would reduce our ability to distinguish the effects of higher versus lower potency products (BonnMiller et al. 2017; Vandrey et al. 2015). We attempted to mitigate this issue with our binning approach and by cutting any observations reporting THC or CBD levels exceeding 30\%/dry wt. Future research would benefit from independent product testing rather than relying on user reporting based on product labels. Despite these limitations, the current finding of a dose-response effect, particularly for flower with higher THC levels, is consistent with the results of several clinical trials (Childs et al. 2017; Tambaro and Bortolato 2012) and suggests that cannabinoid contents is a major factor in Cannabis' potential anxiolytic effects.

\section{Conclusions}

In conclusion, while the clinical drawbacks of using cannabis can include the potential for dependence and addiction and increased risks of motor vehicle accidents, psychotic experiences, and short-term cognitive impairment (National Academies of Sciences, E. and M 2017; Nugent et al. 2017), the side effects reported in the current study were relatively less severe than the more serious medical and sometimes societal problems caused by some conventional prescription (e.g., benzodiazepines and barbiturates) and nonprescription (e.g., alcohol) drugs most used for treating common forms of distress 
(Griswold et al. 2018; Man et al. 2015; Stahre and Simon 2010). Our findings suggest that self-directed use of Cannabis flower, especially that with higher THC levels, is associated with significant improvements in at least short-term feelings of distress in many users, likely a contributing factor to its widespread popularity and consumption in the U.S.

\section{Supplementary Information}

The online version contains supplementary material available at https://doi. org/10.1186/s42238-020-00051-z.

\section{Additional file 1: Supplemental Table 1. Session product}

characteristics' effects on symptom relief when using inhaled, dried Cannabis flower and interacting continuous THC and CBD measures with product characteristics and session length.

\section{Additional file 2: Supplemental Table 2. Session product}

characteristics' effects on symptom relief when using inhaled, dried Cannabis flower subsampling by the number of sessions entered by users.

\section{Additional file 3: Supplemental Table 3. Session product}

characteristics effects on symptom relief when using inhaled, dried

Cannabis flower by time to reported relief.

Additional file 4: Supplemental Table 4. Session product characteristics' effects on side effects when using inhaled, dried Cannabis flower and treating cannabinoid measurements as continuous variables.

\section{Abbreviations}

SSRI: Selective serotonin reuptake inhibitors; SNRI: Serotonin-norepinephrine reuptake inhibitors; TCA: Tricyclic antidepressant; MAOI: Monoamine oxidase inhibitors; $\mathrm{CB}_{1}$ : Cannabinoid receptor 1; $\mathrm{CB}_{2}$ : Cannabinoid receptor 2

\section{Acknowledgements}

We thank all the anonymous Cannabis users that contributed to this research.

\section{Authors' contributions \\ JMV, SSS, and XL conceived the study. FB, KK, and BH independently designed and developed the ReleafApp ${ }^{\mathrm{TM}}$ and server infrastructure as part of their effort to help create an education tool for medical cannabis patients. SSS organized and cleaned the data. XL conducted the analyses. JMV, SSS, $J P D$, and $X L$ drafted the manuscript. All authors contributed substantially to its intellectual content and revision and approved of the final draft submitted.}

\section{Funding}

This research was supported in part by the University of New Mexico Medical Cannabis Research Fund in that donations by unrelated parties to the Medical Cannabis Research Fund were used to provide graduate student funding to Jegason Diviant.

\section{Availability of data and materials}

Data are available from the authors upon reasonable request and with permission of MoreBetter, Ltd., the owners of the Releaf App, and the associated data. The agreement among the authors is nonexclusive and MoreBetter, Ltd. is free to enter into agreements with other researchers.

\section{Ethics approval and consent to participate}

This study was approved by the University of New Mexico Institutional Review Board. All participants consented to have their anonymized recorded data statistically analyzed through the Releaf App Privacy Policy, which must be accepted before any cannabis use sessions can be recorded.

\section{Consent for publication}

All participants consented for their anonymous data to be disseminated in aggregate form.

\section{Competing interests}

JMV is the Director of the University of New Mexico Medical Cannabis Research Fund, while the other authors are affiliated researchers. FB, KK, and $\mathrm{BH}$ developed the Releaf App and own MoreBetter, Ltd. The Releaf App generates revenue through allowing dispensaries to list their specific product offerings in the app, but no compensation is generated for users or the app owners through individual session entries. Publication of this article has no direct effect on compensation for MoreBetter, Ltd., or any of the authors beyond any increase visibility generated by the publication itself. The authors' declare that they have no competing interests.

\section{Author details}

${ }^{1}$ Department of Economics, University of New Mexico, Albuquerque, USA.

${ }^{2}$ Department of Psychology, University of New Mexico, Albuquerque, USA.

${ }^{3}$ MoreBetter, Ltd., Washington, USA.

Received: 1 March 2020 Accepted: 13 November 2020

Published online: 09 December 2020

\section{References}

American Psychological Association. Stress in America: the state of our nation. In: Stress in America Survey; 2017.

Andrade AK, Renda B, Murray JE. Cannabinoids, interoception, and anxiety. Pharmacol Biochem Behav. 2019. https://doi.org/10.1016/j.pbb.2019.03.006.

Bachhuber MA, Saloner B, Cunningham CO, Barry CL. Medical cannabis laws and opioid analgesic overdose mortality in the United States, 1999-2010. JAMA Intern Med. 2014;174(10):1668-73. https://doi.org/10.1001/jamainternmed. 2014.4005 .

Bahi A, Al Mansouri S, Al Memari E, Al Ameri M, Nurulain SM, Ojha S. $\beta$ Caryophyllene, a CB2 receptor agonist produces multiple behavioral changes relevant to anxiety and depression in mice. Physiol Behav. 2014. https://doi. org/10.1016/j.physbeh.2014.06.003.

Bandelow B, Michaelis S. Epidemiology of anxiety disorders in the 21st century. Dialogues Clin Neurosci. 2015;17(3):327-35. https://doi.org/10.31887/DCNS. 2015.17.3/bbandelow.

Bandelow B, Michaelis S, Wedekind D. Treatment of anxiety disorders. Dialogues Clin Neurosci. 2017. https://doi.org/10.4324/9780203728215-32.

Bonaccorso S, Ricciardi A, Zangani C, Chiappini S, Schifano F. Cannabidiol (CBD) use in psychiatric disorders: a systematic review. NeuroToxicology. 2019. https://doi.org/10.1016/j.neuro.2019.08.002.

Bonn-Miller MO, Loflin MJE, Thomas BF, Marcu JP, Hyke T, Vandrey R. Labeling accuracy of cannabidiol extracts sold online. JAMA. 2017. https://doi.org/10. 1001/jama.2017.11909.

Bradford AC, Bradford WD. Medical marijuana laws reduce prescription medication use in medicare part d. Health Aff. 2016;35(7):1230-6. https://doi. org/10.1377/hlthaff.2015.1661.

Buchanan KL. Stress and the evolution of condition-dependent signals. Trends Ecol Evol. 2000. https://doi.org/10.1016/S0169-5347(99)01812-1.

Carvalho-Freitas MIR, Costa M. Anxiolytic and Sedative effects of extracts and essential oil from Citrus aurantium L. Biol Pharm Bull. 2002. https://doi.org/10. 1248/bpb.25.1629.

Childs E, Lutz JA, de Wit H. Dose-related effects of delta-9-THC on emotional responses to acute psychosocial stress. Drug Alcohol Depend. 2017;177:13644. https://doi.org/10.1016/j.drugalcdep.2017.03.030.

Crippa JA, Zuardi AW, Martín-Santos R, Bhattacharyya S, Atakan Z, McGuire P, Fusar-Poli P. Cannabis and anxiety: a critical review of the evidence. Hum Psychopharmacol. 2009. https://doi.org/10.1002/hup.1048.

Cuttler C, Spradlin A, McLaughlin RJ. A naturalistic examination of the perceived effects of cannabis on negative affect. J Affect Disord. 2018. https://doi.org/ 10.1016/j.jad.2018.04.054.

De Almeida AAC, De Carvalho RBF, Silva OA, De Sousa DP, De Freitas RM. Potential antioxidant and anxiolytic effects of $(+)$-limonene epoxide in mice after marble-burying test. Pharmacol Biochem Behav. 2014. https://doi.org/ 10.1016/j.pbb.2014.01.006

De Heer EW, Gerrits MMJG, Beekman ATF, Dekker J, Van Marwijk HWJ, De Waal MWM, et al. The Association of depression and anxiety with pain: a study from NESDA. PLoS One. 2014;9(10):1-11. https://doi.org/10.1371/journal.pone.0106907.

De Moraes Pultrini A, Almeida Galindo L, Costa M. Effects of the essential oil from Citrus aurantium L. in experimental anxiety models in mice. Life Sci. 2006. https://doi.org/10.1016/j.lfs.2005.08.004. 
Dodds TJ. Prescribed benzodiazepines and suicide risk: a review of the literature. Prim Care Companion J Clin Psychiatry. 2017. https://doi.org/10.4088/PCC. $16 r 02037$.

Ferber SG, Namdar D, Hen-Shoval D, Eger G, Koltai H, Shoval G, et al. The "entourage effect": terpenes coupled with cannabinoids for the treatment of mood disorders and anxiety disorders. Curr Neuropharmacol. 2019. https:// doi.org/10.2174/1570159x17666190903103923.

Franco L, Blanck TJJ, Dugan K, Kline R, Shanmugam G, Galotti A, et al. Both lavender fleur oil and unscented oil aromatherapy reduce preoperative anxiety in breast surgery patients: a randomized trial. J Clin Anesth. 2016. https://doi.org/10.1016/j.jclinane.2016.02.032.

Galdino PM, Nascimento MVM, Florentino IF, Lino RC, Fajemiroye JO, Chaibub BA, et al. The anxiolytic-like effect of an essential oil derived from Spiranthera odoratissima A. St. Hil. leaves and its major component, $\beta$-caryophyllene, in male mice. Prog Neuro-Psychopharmacol Biol Psychiatry. 2012; https://doi. org/10.1016/j.pnpbp.2012.04.012.

Gallagher MW, Bentley KH, Barlow DH. Perceived control and vulnerability to anxiety disorders: a meta-analytic review. Cogn Ther Res. 2014. https://doi. org/10.1007/s10608-014-9624-x.

Gallup 2019 global emotions report-Gallup. (2019). Retrieved June 10, 2019, from https://www.gallup.com/analytics/248906/gallup-global-emotionsreport-2019.aspx.

Griswold MG, Fullman N, Hawley C, Arian N, Zimsen SRM, Tymeson HD, et al. Alcohol use and burden for 195 countries and territories, 1990-2016: a systematic analysis for the Global Burden of Disease Study 2016. Lancet. 2018. https://doi.org/10.1016/S0140-6736(18)31310-2.

Gureje O. Comorbidity of Pain and Anxiety Disorders; 2008.

Harada H, Kashiwadani H, Kanmura Y, Kuwaki T. Linalool odor-induced anxiolytic effects in mice. Front Behav Neurosci. 2018. https://doi.org/10.3389/fnbeh. 2018.00241.

Hill EL, Gallopin T, Férézou I, Cauli B, Rossier J, Schweitzer P, Lambolez B. Functional CB1 receptors are broadly expressed in neocortical GABAergic and glutamatergic neurons. J Neurophysiol. 2007. https://doi.org/10.1152/jn. 00603.2006.

Kamal BS, Kamal F, Lantela DE. Cannabis and the anxiety of fragmentation-a systems approach for finding an anxiolytic cannabis chemotype. Front Neurosci. 2018. https://doi.org/10.3389/fnins.2018.00730.

Kasten CR, Zhang Y, Boehm SL. Acute cannabinoids produce robust anxiety-like and locomotor effects in mice, but long-term consequences are age- and sex-dependent. Front Behav Neurosci. 2019. https://doi.org/10.3389/fnbeh. 2019.00032.

Kurlawala Z, Roberts JA, McMillan JD, Friedland RP. Diazepam toxicity presenting as a dementia disorder. J Alzheimers Dis. 2018. https://doi.org/10.3233/JAD180745.

Lima NGPB, De Sousa DP, Pimenta FCF, Alves MF, De Souza FS, MacEdo RO, et al. Anxiolytic-like activity and GC-MS analysis of (R)-(+)-limonene fragrance, a natural compound found in foods and plants. Pharmacol Biochem Behav. 2013. https://doi.org/10.1016/j.pbb.2012.09.005.

Linck VM, da Silva AL, Figueiró M, Caramão EB, Moreno PRH, Elisabetsky E. Effects of inhaled Linalool in anxiety, social interaction and aggressive behavior in mice. Phytomedicine. 2010. https://doi.org/10.1016/j.phymed.2009.10.002.

Man H, Lai X, Cleary M, Sitharthan T, Hunt GE. Prevalence of comorbid substance use, anxiety and mood disorders in epidemiological surveys, 1990 - 2014: a systematic review and meta-analysis. Drug Alcohol Depend. 2015;154:1-13. https://doi.org/10.1016/j.drugalcdep.2015.05.031.

Müller-Oerlinghausen B, Berghöfer A. Antidepressants and suicidal risk. J Clin Psychiatry. 1999;60(Suppl 2):94-9.

National Academies of Sciences, E. and M. The health effects of cannabis and cannabinoids: the current state of evidence and recommendations for research (2017). In: Health effects cannabis cannabinoids; 2017. https://doi. org/10.17226/24625.

Nugent SM, Morasco BJ, O'Neil ME, Freeman M, Low A, Kondo K, et al. The effects of cannabis among adults with chronic painandan overview of general harms a systematic review. Ann Intern Med. 2017;167(5):319-31. https://doi.org/10.7326/M17-0155.

Orsolini L, Chiappini S, Volpe U, De Berardis D, Latini R, Papanti GD, Corkery JM. Use of medicinal cannabis and synthetic cannabinoids in post-traumatic stress disorder (PTSD): a systematic review. Medicina (Lithuania). 2019. https://doi.org/10.3390/medicina55090525.

Pertwee RG, Ross RA. Cannabinoid receptors and their ligands. Prostaglandins Leukot Essent Fatty Acids. 2002. https://doi.org/10.1054/plef.2001.0341.
Piomelli D, Russo EB. The Cannabis sativa versus Cannabis indica debate: an interview with Ethan Russo, MD. Cannabis Cannabinoid Res. 2016. https://doi. org/10.1089/can.2015.29003.ebr.

Piper BJ, Dekeuster RM, Beals ML, Cobb CM, Burchman CA, Perkinson L, et al. Substitution of medical cannabis for pharmaceutical agents for pain, anxiety, and sleep. J Psychopharmacol. 2017. https://doi.org/10.1177/ 0269881117699616.

Powell D, Pacula RL, Jacobson M. Do medical marijuana laws reduce addictions and deaths related to pain killers? J Health Econ. 2018. https://doi.org/10. 1016/j.jhealeco.2017.12.007

Read J, Williams J. Adverse effects of antidepressants reported by a large international cohort: emotional blunting, suicidality, and withdrawal effects. Curr Drug Saf. 2018. https://doi.org/10.2174/ 1574886313666180605095130.

Releaf App | Cannabis Treatment tracking \& research. (2019). Retrieved June 10, 2019, from https://releafapp.com/.

Rey AA, Purrio M, Viveros M, Lutz B. Biphasic effects of cannabinoids in anxiety responses: $C B 1$ and $G A B A B$ receptors in the balance of GABAergic and glutamatergic neurotransmission. Neuropsychopharmacology; 2012;37(12): 2624-34. https://doi.org/10.1038/npp.2012.123.

Ross EM. Handbook of psychotropic herbs: a scientific analysis of herbal remedies for psychiatric conditions. Nutr Clin Care. 2003. https://doi.org/10.1046/j.15235408.2001.00147.x.

Rubino T, Guidali C, Vigano D, Realini N, Valenti M, Massi P, Parolaro D. CB1 receptor stimulation in specific brain areas differently modulate anxiety-related behaviour. Neuropharmacology. 2008;54:151-60. https:// doi.org/10.1016/j.neuropharm.2007.06.024.

Russo EB. Taming THC: potential cannabis synergy and phytocannabinoidterpenoid entourage effects. Br J Pharmacol. 2011. https://doi.org/10.1111/j. 1476-5381.2011.01238.x.

Sarris J, Sinclair J, Karamacoska D, Davidson M, Firth J. Medicinal cannabis for psychiatric disorders: a clinically-focused systematic review. BMC Psychiatry. 2020. https://doi.org/10.1186/s12888-019-2409-8.

Schier A, Ribeiro N, Coutinho D, Machado S, Arias-Carrion O, Crippa J, et al. Antidepressant-like and anxiolytic-like effects of cannabidiol: a chemical compound of Cannabis sativa. CNS Neurol Disord Drug Targets. 2014. https://doi.org/10.2174/1871527313666140612114838.

Shalit N, Lev-Ran S. Does cannabis use increase anxiety disorders? A literature review. Curr Opin Psychiatry. 2020. https://doi.org/10.1097/YCO. 0000000000000560.

Slee A, Nazareth I, Bondaronek P, Liu Y, Cheng Z, Freemantle N. Pharmacological treatments for generalised anxiety disorder: a systematic review and network meta-analysis. 2019;393. https://doi.org/10.1016/S0140-6736(18)31793-8.

Souto-Maior FN, De Carvalho FLD, De Morais LCSL, Netto SM, De Sousa DP, De Almeida RN. Anxiolytic-like effects of inhaled linalool oxide in experimental mouse anxiety models. Pharmacol Biochem Behav. 2011. https://doi.org/10. 1016/j.pbb.2011.08.029.

Stahre M, Simon M. Alcohol-related deaths and hospitalizations by race, gender, and age in California. Open Epidemiol J. 2010. https://doi.org/10.2174/ 1874297101003010003.

Stith SS, Vigil JM. Federal barriers to Cannabis research. Science. 2016;352:1182.

Stith SS, Vigil JM, Adams IM, Reeve AP. Effects of legal access to cannabis on scheduled ii-v drug prescriptions. J Am Med Dir Assoc. 2018a. https://doi. org/10.1016/j.jamda.2017.07.017.

Stith SS, Vigil JM, Brockelman F, Keeling K, Hall B. Patient-reported symptom relief following medical cannabis consumption. Front Pharmacol. 2018b. https:// doi.org/10.3389/fphar.2018.00916.

Stith SS, Vigil JM, Brockelman F, Keeling K, Hall B. The association between cannabis product characteristics and symptom relief. Sci Rep. 2019;9(1):1-8. https://doi.org/10.1038/s41598-019-39462-1.

Tambaro S, Bortolato M. Cannabinoid-related agents in the treatment of anxiety disorders: current knowledge and future perspectives. Recent Pat CNS Drug Discov. 2012. https://doi.org/10.2174/157488912798842269.

Van Ameringen M, Zhang J, Patterson B, Turna J. The role of cannabis in treating anxiety: an update. Curr Opin Psychiatry. 2020. https://doi.org/10.1097/YCO. 0000000000000566 .

Vandrey R, Raber JC, Raber ME, Douglass B, Miller C, Bonn-Miller MO. Cannabinoid dose and label accuracy in edible medical cannabis products. JAMA. 2015. https://doi.org/10.1001/jama.2015.6613.

Vigil J, Stith S, Diviant J, Brockelman F, Keeling K, Hall B. Effectiveness of raw, natural medical cannabis flower for treating insomnia under naturalistic 
conditions. Medicines. 2018;5(3):75. https://doi.org/10.3390/ medicines5030075

Vigil JM, Stith SS, Adams IM, Reeve AP. Associations between medical cannabis and prescription opioid use in chronic pain patients: a preliminary cohort study. PLoS One. 2017;12(11):1-13. https://doi.org/10.1371/journal.pone. 0187795.

Vigil JM. A socio-relational framework of sex differences in the expression of emotion. Behav Brain Sci. 2009. https://doi.org/10.1017/S0140525X09991075.

Viñals X, Moreno E, Lanfumey L, Cordomí A, Pastor A, De La Torre R, et al. Cognitive impairment induced by delta9- tetrahydrocannabinol occurs through heteromers between cannabinoid CB1 and serotonin 5-HT2A receptors. PLoS Biol. 2015. https://doi.org/10.1371/journal.pbio.1002194.

Wen $\mathrm{H}$, Hockenberry JM. Association of medical and adult-use marijuana laws with opioid prescribing for medicaid enrollees. JAMA Intern Med. 2018. https://doi.org/10.1001/jamainternmed.2018.1007.

Wick JY. The history of benzodiazepines. Consult Pharm. 2013. https://doi.org/10. 4140/TCP.n.2013.538.

\section{Publisher's Note}

Springer Nature remains neutral with regard to jurisdictional claims in published maps and institutional affiliations.

Ready to submit your research? Choose BMC and benefit from:

- fast, convenient online submission

- thorough peer review by experienced researchers in your field

- rapid publication on acceptance

- support for research data, including large and complex data types

- gold Open Access which fosters wider collaboration and increased citations

- maximum visibility for your research: over $100 \mathrm{M}$ website views per year

At BMC, research is always in progress.

Learn more biomedcentral.com/submissions 